\title{
Mesotelioma peritoneal: una presentación clínica inusual en un paciente sin exposición al asbesto
}

\author{
J. PONCE LORENZO, A. GIMÉNEZ ORTIZ, F. APARISI APARISI, T. FLEITAS \\ KANONNIKOFF, J. MONTALAR SALCEDO
}

Servicio de Oncología Médica. Hospital Universitario La Fe. Valencia

PERITONEAL MESOTHELIOMA: AN INUSUAL CLINICAL PRESENTATION IN A PATIENT WITHOUT EXPOSURE TO ASBESTOS

\begin{abstract}
RESUMEN
El mesotelioma maligno es un tumor insidioso que se origina de las superficies mesoteliales de las cavidades pleurales y peritoneales, la túnica vaginal o el pericardio. La principal causa relacionada es la exposición al asbesto.

Presentamos el caso de un mesotelioma maligno peritoneal en un varón de 64 años, sin hábitos tóxicos ni exposición al asbesto, que debutó con síndrome constitucional y derrame pleural derecho. La tomografía por emisión de positrones combinada con tomografía axial computerizada (PET-TAC) fue de especial utilidad demostrando un intenso hipermetabolismo en peritoneo con extensión a pared torácica. El paciente inició tratamiento con quimioterapia sistémica, esquema cisplatino-pemetrexed, alcanzando una respuesta parcial tras los 3 ciclos pero con una evolución tórpida secundaria a toxicidad del tratamiento citostático.
\end{abstract}

PALABRAS CLAVE: Mesotelioma peritoneal. PET. Quimioterapia. Cisplatino. Pemetrexed.

\begin{abstract}
Malignant mesothelioma is an insidious neoplasm arising from the mesotelial surfaces, of the pleural and peritoneal cavities, the tunica vaginalis, or the pericardium. The predominant cause is inhalation exposure to asbestos.

We present a rare case of primary malignant mesothelioma of the peritoneum in a 64 year old man without history of inhalation exposure to asbestos. The initial symptoms were constitutional syndrome and right pleural effusion. Positron emission tomography combined with computed tomography (PET/TC) was useful for a supporting diagnosis and to determine the extension. The patient received treatment with systemic palliative chemotherapy, cisplatin-pemetrexed. After three cycles, partial response was observed, but the evolution was fatal due to secondary toxicity of chemotherapy.
\end{abstract}

KEY WORDS: Peritoneal mesothelioma. PET. Chemotherapy. Cisplatin. Pemetrexed.

Ponce Lorenzo J, Giménez Ortiz A, Aparisi Aparisi F, Fleitas Kanonnikoff T, Montalar Salcedo J. Mesotelioma peritoneal: una presentación clínica inusual en un paciente sin exposición al asbesto. An Med Interna (Madrid) 2007; 24: 81-83.

\section{INTRODUCCIÓN}

El mesotelioma maligno es un tumor poco frecuente que se origina de las superficies mesoteliales tales como la pleura, la cavidad peritoneal, la túnica vaginal o el pericardio. El $80 \%$ se originan en la pleura y el $70 \%$ se relacionan con la exposición al asbesto en el ámbito laboral. El mesotelioma peritoneal es una patología infrecuente, de difícil diagnóstico clínico y radiológico y cuyo manejo terapéutico es multimodal.

A continuación presentamos el caso de un varón de 64 años diagnosticado de mesotelioma peritoneal avanzado que debutó de un modo inusual, lo que dificultó todavía más el diagnóstico, y en el cual el PET-TAC fue de especial interés.

\section{CASO APORTADO}

Varón de 64 años, sin hábitos tóxicos, sin antecedentes familiares de cáncer y sin exposición al asbesto en el ámbito laboral, que consulta en octubre 2005 por un cuadro constitucional con astenia, anorexia y leve pérdida de peso de 3 meses de evolución. No tomaba medicación de forma habitual.

En la exploración se objetivó una hipoventilación de los dos tercios inferiores del hemitórax derecho y en la radiografía de tórax apareció un importante derrame pleural derecho, sin signos de con-

Trabajo aceptado: 4 de septiembre de 2006

Correspondencia: Hospital Universitario La Fe. Servicio de Oncología Médica. Avda. Campanar 21. Planta 11 Pabellón Central. 46009 Valencia. e-mail: joseponcelorenzo@hotmail.com 


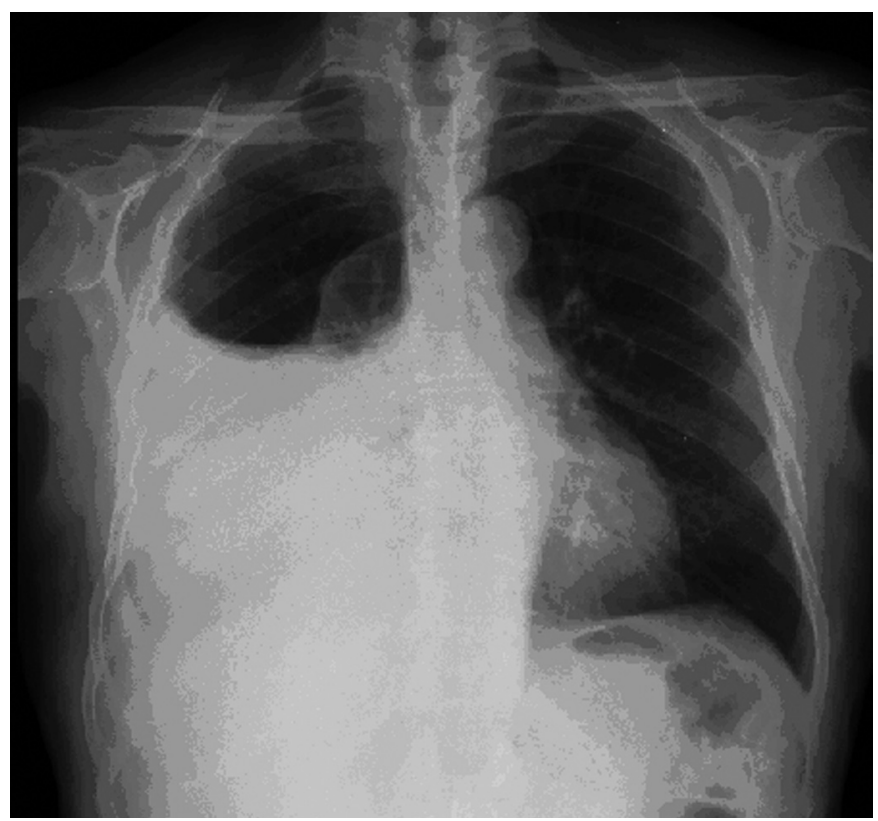

Fig. 1. Radiografía de tórax.

densación parenquimatosa (Fig. 1). No se hallaron resultados patológicos en la bioquímica ni en el hemograma en sangre.

Se realizaron a continuación una broncoscopia, y dos toracoscopias seriadas sin objetivarse hallazgos patológicos (no se observaron lesiones sugerentes de malignidad). Las características del líquido pleural (LP), de color claro-amarillento, fueron compatibles con un exudado: Glucosa $40 \mathrm{mg} / \mathrm{dl}, \mathrm{LDH}(\mathrm{LP}) / \mathrm{LDH}$ (suero) > 0,6 y proteínas (LP)/proteínas (suero) $>0,5$. No se objetivaron células malignas en la citología del mismo.

En la TAC toraco-abdominal se halló un engrosamiento difuso de la crura diafragmática derecha en toda su circunferencia, mostrando además afectación de la pared torácica anterior, con afectación del músculo recto anterior y de la pared abdominal antero-lateral derecha. También se objetivaron el derrame pleural derecho y mínimo líquido libre ascítico.

Se realizó a continuación una biopsia eco-dirigida de la pared abdominal anterior, en la cual se objetivó una proliferación celular mesotelial de acusada intensidad compatible con mesotelioma subtipo epitelial. Las celulas tumorales expresaban en la inmunohistoquimia CKAE-1-AE3, CK-7 y calretinina (nuclear y citoplasmática) siendo negativas CK-20 y CEA. Se descartaron otros tipos de tumores como carcinoma, linfoma, sarcoma o melanoma.

Se solicitó una PET-TAC que demostró un intenso hipermetabolismo en peritoneo (SUV máx $=13,9 \mathrm{~g} / \mathrm{ml}$ ), a nivel perihepático con extensión a pared torácica e hipocondrio izquierdo anterior al bazo, compatible con malignidad (Figs. 2a y 2b). Se visualizó además un gran derrrame pleural derecho asociado a una atelectasia, ambos ametabólicos.

Finalmente, se alcanzó el diagnóstico definitivo de mesotelioma maligno de origen peritoneal con afectación de pared torácica, avanzado e irresecable.

Descartada pues la cirugía por la extensión de la enfermedad se decidió iniciar tratamiento quimioterápico sistémico con el esquema cisplatino-pemetrexed. Tras tres ciclos completos se evidenció una respuesta parcial radiológica de la enfermedad. Sin embargo la evolución fue tórpida, con diarrea, insuficiencia renal y neumonía en el contexto de neutropenia grado 4 , secundarios al tratamiento citostático. A pesar del tratamiento de soporte con fluidoterapia intravenosa y antibioterapia de amplio espectro el paciente no mejoró, falleciendo finalmente en marzo del 2006.

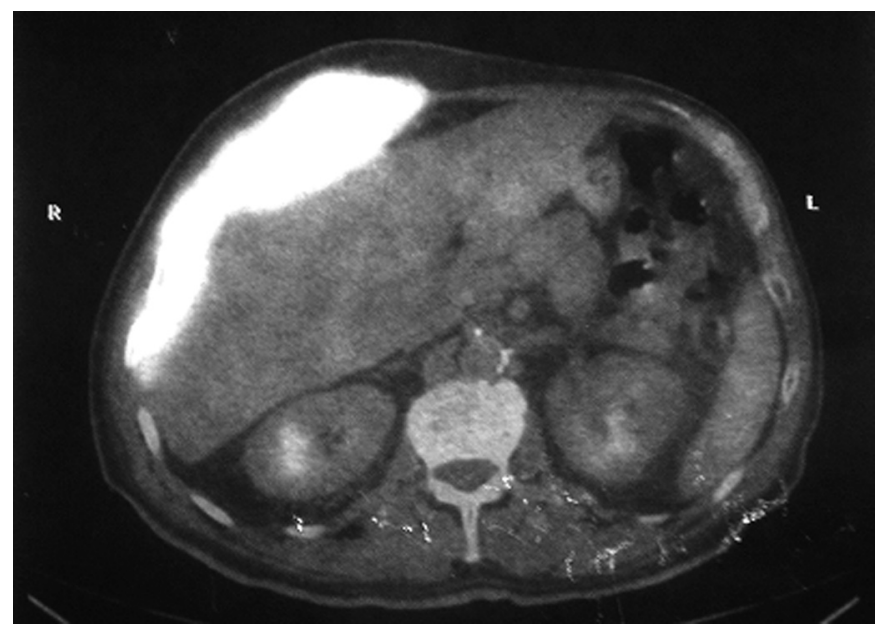

Fig. 2a. PET-TAC: hipermetabolismo en peritoneo perihepático.

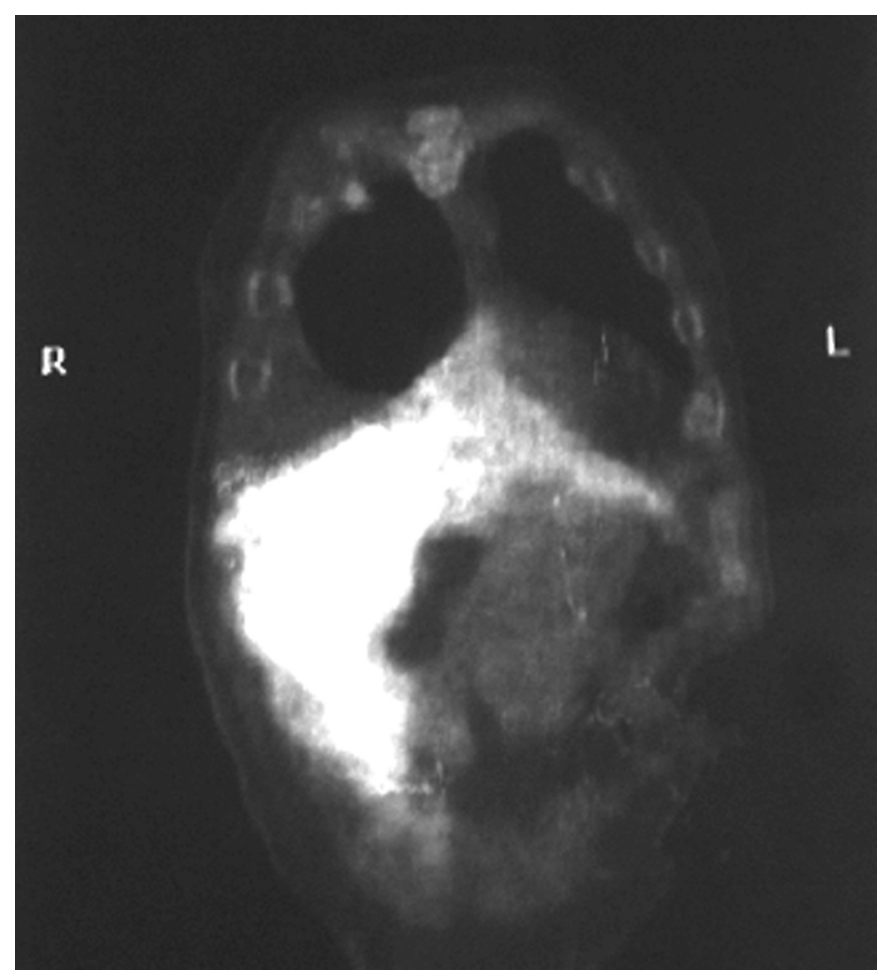

Fig. 2b. PET-TEC: hipermetabolismo en peritoneo con extensión supradiafragmática.

\section{DISCUSIÓN}

El mesotelioma maligno es una neoplasia insidiosa poco frecuente que surge de las superficies mesoteliales, ya sea de la pleura, peritoneo, túnica vaginal o pericardio. El $80 \%$ de los casos son de origen pleural y el $70 \%$ de los casos se relacionan con la exposición en el ámbito laboral al asbesto [1]. De hecho, los trabajadores expuestos al asbesto tienen un riesgo del $10 \%$ de diagnosticarse de un mesotelioma maligno a lo largo de su vida. La latencia entre la exposición y el riesgo de padecer la enfermedad es larga, aproximadamente de 30 a 40 años (2,3). 
Histológicamente los mesoteliomas se clasifican en tres subtipos: epitelial, sarcomatoide y bifásico. La inmunohistoquimia es de especial utilidad, y la presencia de marcadores como la calretinina y la ausencia de otros como el CEA apoyan el diagnóstico de mesotelioma, como en nuestro caso (4)

El mesotelioma peritoneal, al igual que el pleural también tiene una fuerte asociación al asbesto (5). La clínica habitual con la que suele debutar incluye dolor y distensión abdominal, ascitis, masa abdominal o pélvica y trombocitosis (6).

En la mayoría de los pacientes, la enfermedad se manifiesta de forma localmente agresiva con un difícil manejo terapéutico. En las formas localizadas, no avanzadas, el tratamiento es multidisciplinar. A lo largo de las últimas décadas se han combinado diversas estrategias: cirugía sola, cirugía seguido de quimioterapia intraperitoneal y cirugía seguido de quimioterapia sistémica, generalmente con esquemas basados en cisplatino. Aunque no existen estudios comparativos que demuestren una clara superioridad, las series de pacientes tratados con cirugía seguido de quimioterapia intraperito-

\section{Bibliografía}

1. Animan, KH. Natural history and epidemiology of malignant mesotelioma. Chest 1993; 103:373S

2. Selikoff, IJ, Hammond, EC, Seidman, H. Latency of asbestos disease among insulation workers in the United States and Canada. Cancer 1980; 46: 2736

3. Hansen, J, de Klerk, NH, Musk AW, et al. Environmental exposure to crocidolite and mesothelioma. Exposure-response realationships. Am J Respir Crit Care Med 1998; 157: 69.

4. Doglioni, C, Tos, AP, Laurino, L, et al. Calretinin: a novel immunocytochemical marker for mesothelioma. Am J Surg Pathol 1996; 20: 1037.

5. Berry, G, Newhouse, ML, Wagner, JC. Mortality from all cancers of asbestos factory workers in east London. 1933-80. Occup Environ Med 2000; 57: 782.

6. Eltabbakh, GH, Piver, MS, Hempling, RE, et al. Clinical Picture, response to therapy, and survival of women with diffuse malignant pritoneal mesotelioma. J Surg Oncol 1999; 70: 6.

7. Labow, DM, Coit, DG, Kelsen, D, et al. Primary peritoneal mesothelioma: neal alcanzaron mayores supervivencias, de 34 a 58 meses (7-9).

En los pacientes con enfermedad avanzada (irresecable o recidivante),el pronóstico es infausto con supervivencias menores de 1 año. El tratamiento sistémico quimioterápico con cisplatino-pemetrexed ha demostrado un $26 \%$ de respuetas parciales objetivas, con resultados similares tanto en aquellos que debutan en enfermedad avanzada como en los que han recidivado tras la cirugía y la quimioterapia intraperitoneal (10). Otros agentes como paclitaxel, mitomicina, adriamicina o irinotecán han demostrado actividad, sólos o en combinación con cisplatino, con un menor índice de respuestas (6,11-13).

En conclusión, hemos presentado un caso de una patología poco frecuente con un debut clínico inusual, en el que la PET-TAC fue especialmente útil para el diagóstico y extensión de la enfermedad y cuya evolución fue tórpida secundaria al tratamiento a pesar de una respuesta radiológica objetiva. how aggressive should we be? Proc Am Soc Clin Oncol 2004; 23: 345a.

8. Loggie, BW, Fleming, RA, McQuellon, RP, et al. Prospective trial for the treatment of malignant peritoneal mesothelioma. Am Surg 2001; 67: 999.

9. Sugarbaker, PH, Acherman, YI, Gonzalez-Moreno, S, et al. Diagnosis and treatment of peritoneal mesothelioma: The Washington Cancer Institute experience. Semin Oncol 2002; 29: 51.

10. Janne, PA, Wozniak, AJ, Belani, CP, et al. Open-label study of pemetrexed alone or in combination with cisplatin for the treatment of patients with peritoneal mesothelioma: outcomes of an expanded access program. Clin Lung Cancer 2005; 7: 40.

11. Taub, RN, Keohan, ML, Chabot, JC, et al. Peritoneal mesothelioma. Curr Treat Options Oncol 2000; 1: 303.

12. Tsavaris, N, Primikiros, N, Mylonakis, N, et al. Combination chemotherapy with cisplatin and/or doxorubicin in malignant mesothelioma. A retrospective study. Anticancer Res 1997; 17: 3799.

13. Le, DT, Deavers, M, Hunt, K, et al. Cisplatin and irinotecán (CPT-11) for peritoneal mesothelioma. Cancer Invest 2003; $21: 682$. 